\title{
Ku70 is essential for histone deacetylase inhibitor trichostatin A-induced apoptosis
}

\author{
JIN MENG ${ }^{1,2^{*}}$, FENG ZHANG $^{1 *}$, XU-TAO ZHANG ${ }^{1}$, TAO ZHANG $^{3}$, YU-HUA LI ${ }^{1}$, \\ LEI FAN ${ }^{1}$, YANG SUN ${ }^{1}$, HE-LONG ZHANG ${ }^{4}$ and QI-BING MEI ${ }^{1}$ \\ ${ }^{1}$ Key Laboratory of Gastrointestinal Pharmacology of Chinese Materia Medica of The State Administration of \\ Traditional Chinese Medicine, Department of Pharmacology, School of Pharmacy, The Fourth Military Medical University, \\ Xi'an, Shaanxi 710032; ${ }^{2}$ Department of Pharmacy, The 309th Hospital of PLA, Beijing 100091; \\ Departments of ${ }^{3}$ Thoracic Surgery and ${ }^{4}$ Oncology, Tangdu Hospital, The Fourth Military Medical University, \\ Xi'an, Shaanxi 710038, P.R. China
}

Received March 5, 2014; Accepted January 2, 2015

DOI: $10.3892 / \mathrm{mmr} .2015 .3358$

\begin{abstract}
It was previously reported that the histone deacetylase inhibitor (HDACI) trichostatin A (TSA) induced B cell lymphoma 2 (Bcl-2)-associated X protein (Bax)-dependent apoptosis in colorectal cancer (CRC) cells. In addition, Ku70 has been identified as a regulator of apoptosis, the mechanism of which proceeds via interacting with Bax. The aim of the present study was to investigate the role of Ku70 in TSA-induced apoptosis in the CRC cell lines HCT116 and HT29. The results showed that TSA induced the acetylation of Ku70, which was found to be associated with increased apoptosis. In addition, TSA treatment promoted the release of Bax from its complex with Ku70. Bax was then detected to have translocated from the cytoplasm into the mitochondria, while cytochrome $c$ was detected to have translocated from the mitochondria into the cytoplasm. Furthermore, knockdown of Ku70 using small interfering RNA decreased TSA-induced apoptosis as well as downregulated the
\end{abstract}

Correspondence to: Dr Qi-Bing Mei, Key Laboratory of Gastrointestinal Pharmacology of Chinese Materia Medica of The State Administration of Traditional Chinese Medicine, Department of Pharmacology, School of Pharmacy, The Fourth Military Medical University, 17 Changlexi Road, Xi'an, Shaanxi 710032, P.R. China E-mail: mei_qb@163.com

Dr He-Long Zhang, Department of Oncology, Tangdu Hospital, The Fourth Military Medical University, 1 Xinsi Road, Xi'an, Shaanxi 710038, P.R. China

E-mail: cnxazhl@163.com

${ }^{*}$ Contributed equally

Abbreviations: TSA, trichostatin A; CRC, colorectal cancer; HDACI, histone deacetylase inhibitor

Key words: Ku70, trichostatin A, B-cell lymphoma 2-associated X protein, colorectal cancer cells, apoptosis expression of Bax. These effects were rescued through pre-treatment of cells with the proteasome inhibitor MG132. In conclusion, the results of the present study suggested that Ku70 acetylation mediated TSA-induced apoptosis in CRC cells. In addition, $\mathrm{Ku} 70$ was found to be indispensable in TSA-induced apoptosis due to its role in protecting Bax from proteosomal degradation.

\section{Introduction}

Colorectal cancer (CRC) was reported to be the second most prevalent cause of cancer-associated mortality worldwide in 2009 (1). Early-stage colorectal cancer is treatable and may be cured through surgical resection; however, the rate of recurrence remains high (1). Therefore, it is essential to develop novel therapeutic strategies for the treatment of CRC.

Histone deacetylase inhibitors (HDACIs) have an important role in the regulation of genes which are associated with the cell cycle and apoptosis; in addition, HDACIs have been suggested to have promising anti-cancer properties (2-4). Trichostatin A (TSA), a pan-HDAC inhibitor, has been reported to induce cell cycle arrest, promote cell differentiation and apoptosis as well as inhibit metastasis (5) in numerous types of tumor (6). It was previously demonstrated that TSA induced apoptosis in CRC cells through p53-dependent and -independent pathways via B cell lymphoma 2 (Bcl-2)-associated X protein (Bax)-dependent mechanisms (7). Ku70 is an important protein in DNA damage repair, which has also been reported to participate in apoptosis regulation through interacting with Bax in the cytoplasm and inhibiting the translocation of Bax to the mitochondria upon receiving an apoptosis signal (8). In addition, it was reported that acetylation of $\mathrm{Ku} 70$ may disrupt its association with Bax, therefore releasing Bax from the complex and resulting in Bax-dependent mitochondrial apoptosis (9). However, the function of Ku70 in TSA-induced apoptosis of CRC cells remains to be elucidated.

The aim of the present study was to investigate the role of Ku70 in TSA-induced apoptosis in the CRC cell lines HCT116 and HT29. 


\section{Materials and methods}

Cell culture and treatments. The CRC cell lines HCT116 and HT29 (American Type Culture Collection, Manassas, VA, USA) were cultured in Dulbecco's modified Eagle's medium (DMEM; Gibco-BRL, Carlsbad, CA, USA) supplemented with $10 \%$ fetal bovine serum (Gibco-BRL), $10 \mathrm{mg} / \mathrm{ml}$ antibiotics (penicillin and streptomycin; Sigma-Aldrich, St Louis, MO, USA) and $2 \mathrm{mmol} / \mathrm{l} \mathrm{L}$-glutamine at $37^{\circ} \mathrm{C}$ under a humidified atmosphere with $5 \% \mathrm{CO}_{2}$. TSA (Sigma-Aldrich) was dissolved in dimethyl sulfoxide (DMSO; Sigma-Aldrich) to a final concentration of $1.0 \mu \mathrm{M}$, which was used to treat the cells $\left(3 \times 10^{5}\right.$ cells/well in a six-well plate). An equal volume of DMSO was used as vehicle control. The cells were incubated with TSA for $15 \mathrm{~h}$ for Bax detection, and for $24 \mathrm{~h}$ for the detection of the other proteins.

Annexin V-fluorescein isothiocyanate (FITC) and propidium iodide (PI) staining. HCT116 and HT29 cells were cultured and treated with TSA as described above; cells were then digested using trypsin (Gibco-BRL), stained with $5 \mu 1$ Annexin V-FITC (BD Pharmingen, San Diego, CA, USA) and $5 \mu \mathrm{l}$ PI (BD Pharmingen) staining solution in the dark at room temperature (RT) for $15 \mathrm{~min}$. The cell samples were analyzed by flow cytometry using a FACScan station with Cell Quest software (BD Biosciences, Franklin Lakes, NJ, USA) using the fluorescent resolution FL1 and FL2 ranges for Annexin V FITC and PI, respectively.

Western blot analysis and co-immunoprecipitation. Cells were lysed in lysis buffer containing $150 \mathrm{mM} \mathrm{NaCl}, 1 \% \mathrm{NP} 40$, $0.5 \%$ deoxycholic acid, $0.1 \%$ SDS, $50 \mathrm{mM}$ Tris (pH 8.0) (Sigma-Aldrich) and 1:25 protease inhibitor cocktail. For cellular fractionation, cells were washed with phosphate-buffered saline (PBS) and lysed in cytosolic lysis buffer containing $250 \mathrm{mM}$ sucrose, $70 \mathrm{mM} \mathrm{KCl}, 137 \mathrm{mM}$ $\mathrm{NaCl}, 4.3 \mathrm{mM} \mathrm{Na} \mathrm{HPO}_{4}, 1.4 \mathrm{mM} \mathrm{KH_{2 }} \mathrm{PO}_{4}(\mathrm{pH} 7.2)$, $200 \mu \mathrm{g} / \mathrm{ml}$ digitonin, $100 \mathrm{mM}$ phenylmethylsulfonyl fluoride and 1:25 protease inhibitor cocktail for $30 \mathrm{~min}$ on ice. Cells were centrifuged at $16,873 \mathrm{xg}$ for $15 \mathrm{~min}$ at $4^{\circ} \mathrm{C}$. The supernatants were stored as cytosolic protein extract and the pellets were further dissolved with lysis buffer containing $1 \%$ SDS as the mitochondrial fraction. Protein concentrations of the lysates were determined using the Bradford protein assay kit (Bio-Rad Laboratories, Inc., Hercules, CA). In brief, equal amounts of protein $(30 \mu \mathrm{g}$ protein/lane) were separated using SDS-PAGE (5\% stacking gel and 12\% separation gel), transferred to nitrocellulose membranes (Hybond C, GE Healthcare, Little Chalfont, UK). Immunoblots were blocked with 5\% skimmed milk in Tris-buffered saline/Tween 20 $(0.05 \%, \mathrm{v} / \mathrm{v})($ Sigma-Aldrich) for $1 \mathrm{~h}$ at RT. The membranes were then incubated with the following primary antibodies overnight at $4{ }^{\circ} \mathrm{C}$ : Mouse monoclonal anti-poly-adenyl-ribosyl polymerase (PARP; 1:2,000 dilution; cat. no. 556362; BD Pharmingen), rabbit monoclonal anti-cytochrome $c$ (1:1,000 dilution; cat. no. 3895-1; Epitomics, Burlingame, CA, USA), rabbit polyclonal anti-cycloxygenase (COX) IV (1: 500 dilution; cat. no. GTX101499; GeneTex, San Antonio, TX, USA), rabbit monoclonal anti-Bax (1:1,000 dilution; cat. no. 1063-1; Epitomics), rabbit monoclonal anti-Ku70

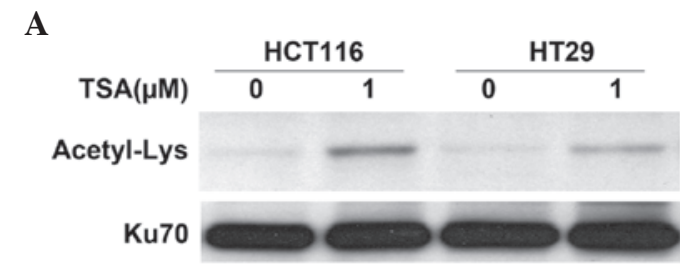

B

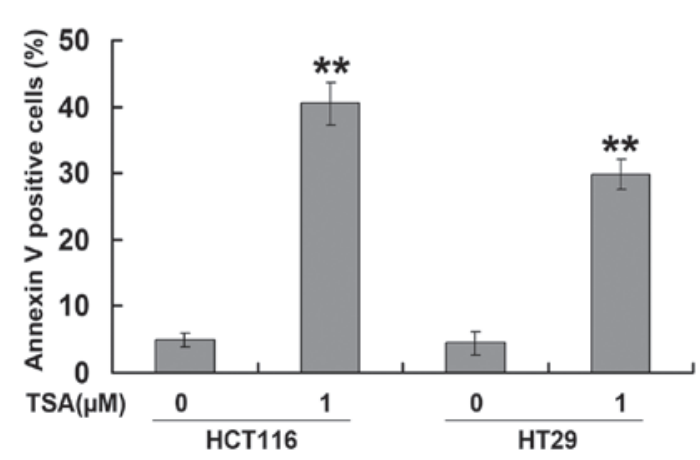

Figure 1. TSA induces Ku70 acetylation and apoptosis in CRC cell lines. HCT116 and HT29 cells were exposed to TSA $(1 \mu \mathrm{M})$ for 24 h. (A) Ku70 acetylation was detected using western blot and immunopreciptation analyses. (B) Apoptosis was analyzed by Annexin V-fluorescein isothiocyanate and propidium iodide staining followed by flow cytometric analysis Percentages of apoptotic cells are indicated by Annexin V-positive cells and presented as the mean \pm standard deviation $(\mathrm{n}=3)$. ${ }^{* *} \mathrm{P}<0.01$ vs. $0 \mu \mathrm{M}$ TSA. TSA, trichostatin A.

(1:1,000; cat. no. 2829-1; Epitomics), rabbit polyclonal anti-active caspase 3 (1:800; cat. no. Ab2302; Abcam, Cambridge, MA, USA) and mouse monoclonal anti-actin (1:5,000 dilution; cat. no. A1978; Sigma-Aldrich). The membranes were then incubated with the corresponding secondary antibodies, including polyclonal goat anti-mouse IgG-H\&L (1:7,000 dilution; cat. no. ab6787; Abcam) and polyclonal goat anti-rabbit IgG-H\&L (1:7,000 dilution; cat. no. ab6721; Abcam) conjugated with horseradish peroxidase at RT for $1 \mathrm{~h}$. Blots were developed using an enhanced chemiluminescence western blotting detection kit (GE Healthcare). For co-immunoprecipitation, HCT116 cells were treated with TSA $(1 \mu \mathrm{M})$ and the cell lysates were precipitated with anti-Ku70 antibodies. The bound proteins were then subjected to SDS-PAGE and blotted for Bax, as previously described (10).

Ku70 small interfering (si)RNA transfection. Ku70 siRNA and scrambled siRNA were purchased from Santa Cruz Biotechnology Inc. A siPORT NeoFX transfection agent (Ambion Life Techonolgies, Grand Island, NY, USA) was used for the siRNA transfection. At $24 \mathrm{~h}$ post-transfection, cells were treated with TSA for a further $24 \mathrm{~h}$. Knockdown of Ku70 expression was confirmed by western blot analysis.

Statistical analysis. All values are presented as the mean \pm standard deviation or a representative of $\geq 3$ independent experiments. Statistical comparisons were performed using the Student's $t$-test. SPSS version 11.0 (SPSS Inc., Chicago, IL, USA) was used to perfom all statistical analyses. $\mathrm{P}<0.05$ was considered to indicate a statistically significant difference between values. 


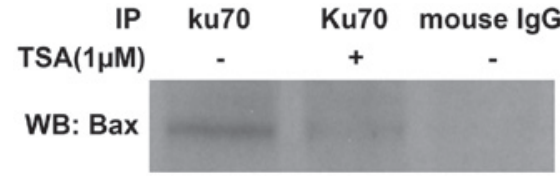

Figure 2. TSA promotes Bax release from its association with Ku70. HCT116 cells were treated with or without TSA $(1 \mu \mathrm{M})$. The interaction between Ku70 and Bax was confirmed by IP with anti-Ku70 antibodies and subsequent western blot analysis of Bax protein expression. Mouse IgG was used as the control. TSA, trichostatin A; Bax, B cell lymphoma 2-associated $\mathrm{X}$ protein; IP, immunoprecipitation; WB, western blot.

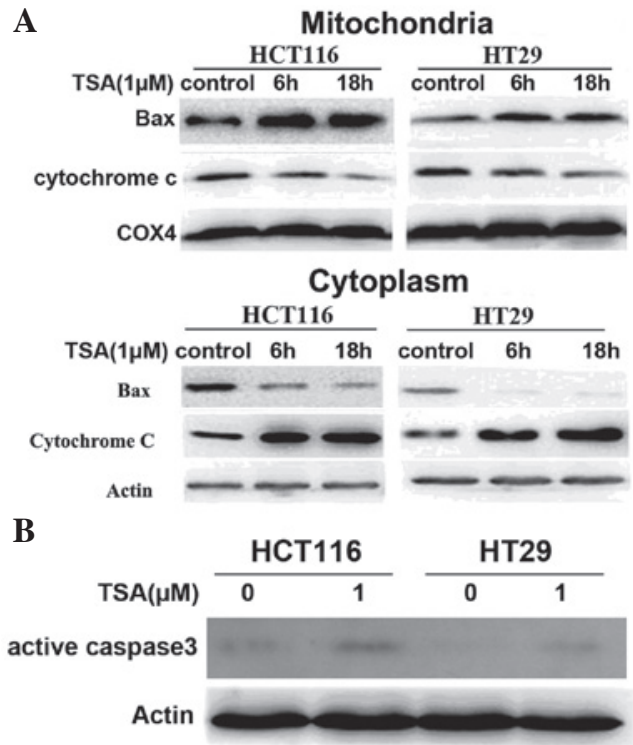

Figure 3. TSA induces translocation of Bax and cytochrome $c$ as well as caspase-3 activation. HCT116 and HT29 cells were exposed to $1 \mu \mathrm{M}$ TSA for 6 and $18 \mathrm{~h}$. (A) Proteins from the cytoplasm and mitochondria were extracted and analyzed using western blotting with anti-Bax and anti-cytochrome $c$ antibodies. Actin and COX4 blots were used as the cytoplasmic and mitochondrial loading controls, respectively. (B) Western blot analysis using anti-active caspase- 3 antibodies was performed to determine active caspase 3 levels in the cytoplasm. Actin was used as the loading control. TSA, trichostatin A; Bax, B cell lymphoma 2 -associated X protein; COX4, cycloxygenase IV.

\section{Results}

TSA induces Ku70 acetylation and apoptosis in CRC cell lines. HCT116 and HT29 cells were exposed to various concentrations of TSA $(0.1,1.0$ and $5.0 \mu \mathrm{M})$ for $24 \mathrm{~h}$. Ku70 acetylation was detected by western blot and immunoprecipitation analysis. As shown in Fig. 1A, TSA induced the acetylation of Ku70 in HCT116 and HT29 cells. In addition, TSA was found to significantly enhance apoptosis, as measured using Annexin-V-FITC/PI staining (Fig. 1B), which was in parallel with changes in Ku70 acetylation levels. These results therefore suggested that Ku70 acetylation was involved in the mechanism of TSA-induced apoptosis in CRC cells.

TSA promotes Bax release from its association with Ku70. As TSA was found to induce Ku70 acetylation, it was investigated whether TSA treatment impaired the interaction between Ku70 and Bax. Co-immunoprecipitation analysis was performed, the results of which revealed a weakened Bax band in TSA-treated cells following protein lysate immunoprecipitation by the $\mathrm{Ku} 70$ antibody compared with that of the untreated Bax band (Fig. 2). These results indicated that Bax was released from its association with Ku70 upon TSA-mediated Ku70 acetylation.

TSA induces Bax translocation from the cytoplasm to the mitochondria and caspase-3 activation. Bax translocation is an early event in Bax-dependent apoptosis, which results in damage of the mitochondrial outer membrane and the release of key apoptosis mediators, such as cytochrome $c$, subsequently leading to the activation of caspase- 9 and the downstream cleavage of caspase-3 $(11,12)$. In the present study, Bax localization in the cytosol and mitochondria was investigated following cell fractionation. This was performed using immunoblotting of lysates of HCT116 and HT29 cells following exposure to $1 \mu \mathrm{M}$ TSA. As shown in Fig. 3A, Bax levels were high in the cytoplasm of control cells and declined following TSA treatment. By contrast, Bax levels in the mitochondria were increased when treated with TSA. In addition, cytochrome $c$ levels in the cytoplasm were increased following TSA treatment, which was accompanied by a decrease in the mitochondrial fraction (Fig. 3A). Furthermore, western blot analysis revealed that active caspase 3 expression was increased following TSA treatment (Fig. 3B), indicating that TSA enhanced Bax translocation from the cytosol into the mitochondria, leading to the release of cytochrome $c$ into the cytosol, which resulted in activation of caspase 3 .

Knockdown of Ku70 via siRNA impairs TSA-induced apoptosis. Ku70 has previously been reported to regulate apoptosis through interacting with cytosolic Bax and inhibiting its translocation to the mitochondria (8). It was therefore hypothesized that decreased Ku70 may increase the release of Bax, which translocates to the mitochondria and initiates apoptosis upon TSA treatment. By contrast, the results of the present study showed that Ku70 siRNA impaired TSA-induced apoptosis in CRC cells, as determined by flow cytometric and western blot analysis (Fig. 4A-C). In addition, this decrease in apoptosis was accompanied with a decline in Bax expression, as detected by western blot analysis (Fig. 4D).

Decreased apoptosis by Ku70 siRNA following TSA treatment is rescued by pre-treating cells with proteasome inhibitor MG132. The decreased Bax protein levels may be due to proteasomal degradation, which increases the instability of the protein. MG132 is a potent proteasome inhibitor, which was found to induce apoptosis in CRC cells in a dose-dependent manner (Fig. 5A). A low dose of MG132 $(0.5 \mu \mathrm{M})$, which was shown to have little effect on apoptosis, was selected for the pre-treatment of HCT116 cells prior to TSA treatment. MG132 pre-treatment rescued the Ku70 siRNA-induced decrease in apoptosis (Fig. 5B and $\mathrm{C}$ ), as well as decreased the protein expression levels of activated caspase 3 (Fig. 5D) and Bax (Fig. 5E). These results demonstrated that Ku70 may have an important role in the protection of Bax against proteasomal degradation.

\section{Discussion}

HDACIs have been reported to be a class of relatively selective candidates of anti-cancer agents, which are thought to 
A

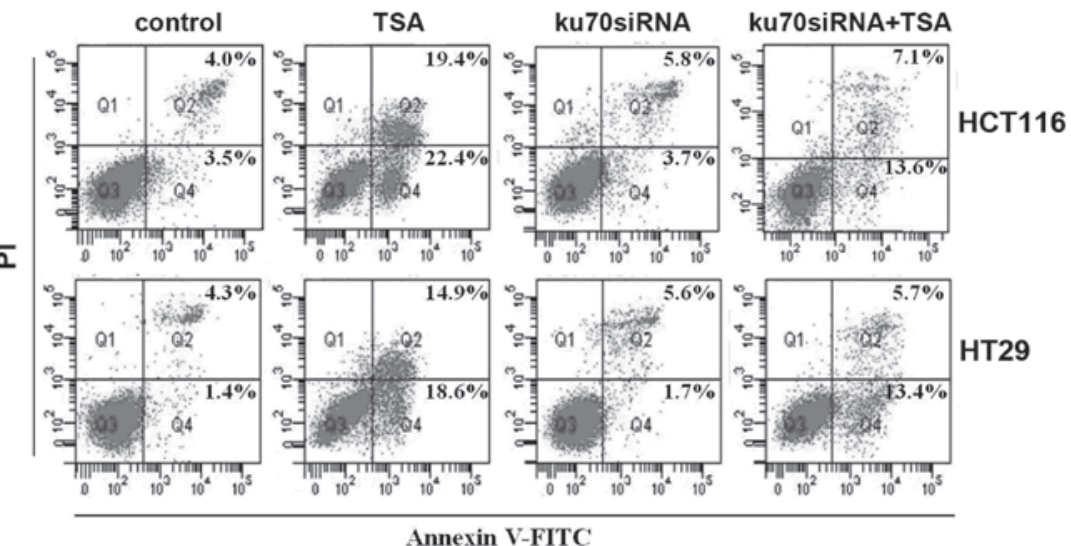

B

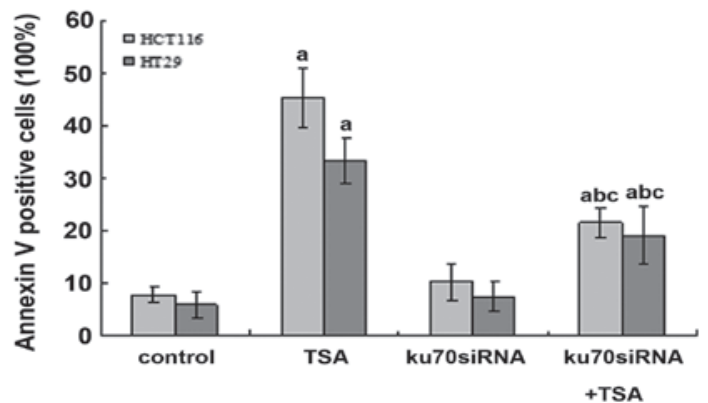

C

HCT116

HT29

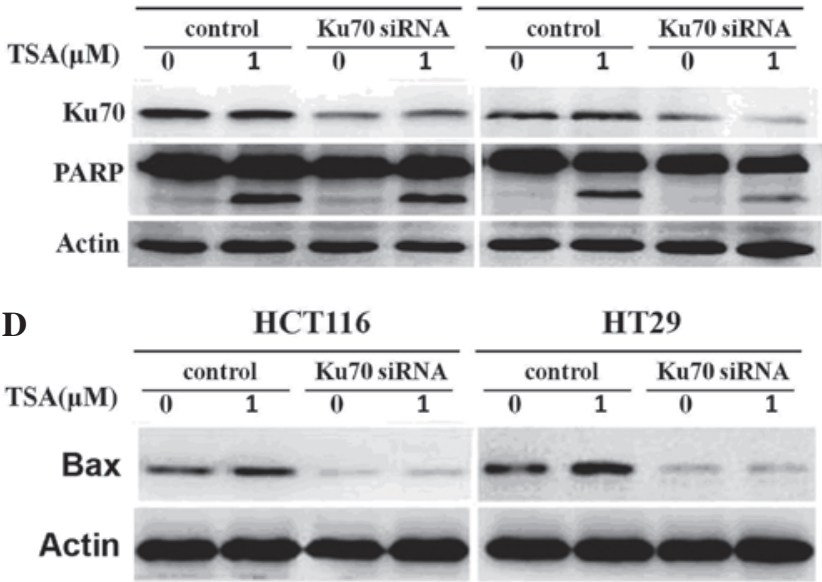

Figure 4. Ku70 depletion impairs TSA-induced apoptosis. Ku70 siRNA and scramble siRNA were transfected to HCT116 and HT29 cells 24 h prior to treatment with TSA for $24 \mathrm{~h}$. (A) Cells were collected and apoptosis was analyzed by Annexin V-FITC and PI staining and flow cytometric analysis. Representative plots of one set of triplicate experiments are shown. (B) Percentages of apoptotic cells are indicated by Annexin V-positive cells and presented as the mean \pm standard deviation $(\mathrm{n}=3)$. ${ }^{\mathrm{a}} \mathrm{P}<0.01$ vs. control, ${ }^{\mathrm{b}} \mathrm{P}<0.01$ vs. Ku70 siRNA-treated cells and ${ }^{\mathrm{C}} \mathrm{P}<0.01$ vs. TSA-treated cells. (C) Ku70 expression, PARP cleavage levels and (D) Bax expression levels were detected using western blot analysis. Actin was used as the loading control. TSA, trichostatin A; siRNA, small interfering RNA; FITC, fluorescein isothiocyanate; PI, propidium iodide; PARP, polyadenylribosyl polymerase; Bax, B cell lymphoma 2-associated $\mathrm{X}$ protein.

induce cell growth arrest and trigger the apoptosis of tumor cells (13). A previous study demonstrated that TSA induced apoptosis in CRC cells via Bax-dependent mechanisms. Bax is a pro-apoptotic protein, which is essential for the initiation of mitochondria-mediated apoptosis, the mechanism of which proceeds through permeabilizing the mitochondrial outer membrane, resulting in cytochrome $c$ release from mitochondria, which then associates with the $47 \mathrm{kDa}$ procaspase-9/apoptotic protease activating factor 1 and activates the caspase cascade, leading to apoptosis (14). It was reported that Bax-initiated apoptosis was suppressed by Ku70, which interacted with Bax and sequestering it from the mitochondria (8).
Ku70 functions as a DNA repair protein in the nucleus and as an anti-apoptotic protein through binding to Bax in the cytoplasm (8). In neuroblastoma cells, increased Ku70 acetylation was found to result in the release of Bax from its complex with Ku70, therefore triggering programmed cell death (15). The results of the present study have shown that Ku70 acetylation and the subsequent release and activation of Bax were also involved in TSA-induced apoptosis in CRC cells; this therefore indicated that this may be an important mechanism by which Bax-dependent apoptosis was mediated.

$\mathrm{Ku} 70$ is known to be a repair protein as well as an inhibitor of apoptosis through its association with Bax (8). The results 
A

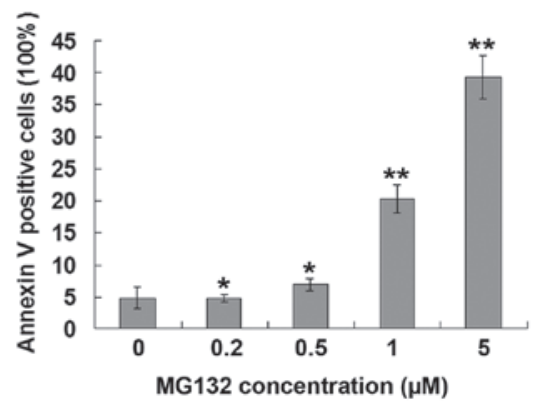

B

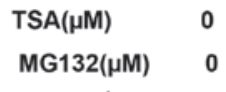

\section{1}

0
1

0.5

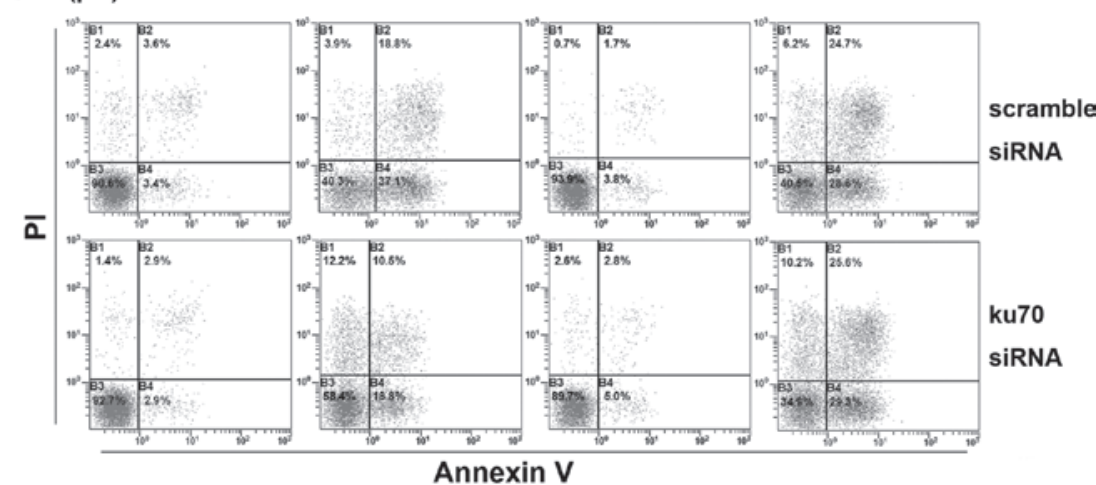

$\mathbf{D}$

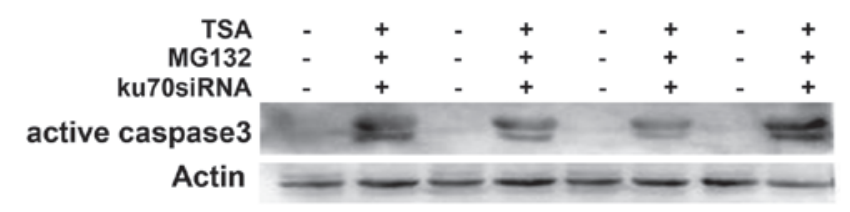

$\mathbf{E}$

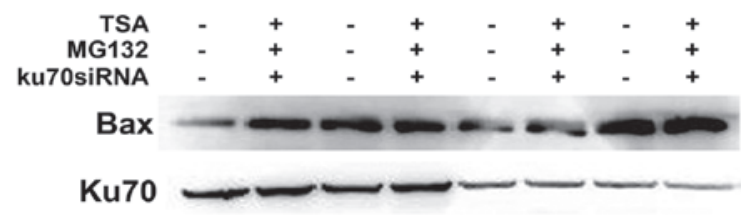

Actin

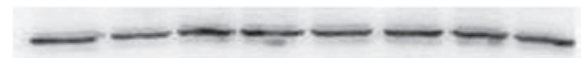

Figure 5. Decreased apoptosis due to Ku70 depletion is rescued by proteosomal inhibitor MG132. (A) Various concentrations of MG132 were used to treat HCT116 cells for $24 \mathrm{~h}$ prior to apoptosis detection by flow cytometry. ${ }^{*} \mathrm{P}>0.05$ and ${ }^{* *} \mathrm{P}<0.01$ vs. control. (B) HCT116 cells were transfected with Ku70 siRNA prior to treatment with $0.5 \mu \mathrm{M}$ MG132 and $1 \mu \mathrm{M}$ TSA. Cells were collected $24 \mathrm{~h}$ following TSA treatment. Apoptosis was detected by flow cytometric analysis. Representative plots of one set of triplicate experiments are shown. (C) Percentages of apoptotic cells as indicated by Annexin V-positive cells. (D) PARP cleavage and activated caspase 3 expression levels were detected using western blot analysis. Actin was used as the loading control. (E) HCT116 cells were treated as described above and cells were collected $15 \mathrm{~h}$ post-TSA incubation. Bax and Ku70 expression were detected using western blot analysis. Actin was used as the loading control. Values are presented as the meant \pm standard deviation $(n=3)$. siRNA, small interfering RNA; TSA, trichostatin A; PI, propidium iodide; PARP, polyadenylribosyl polymerase; Bax, B cell lymphoma 2-associated X protein.

of the present study have also demonstrated that TSA induced cell death in CRC cells through increasing the acetylation of $\mathrm{Ku} 70$, a Bax-binding protein, which therefore promoted Bax release and translocation from the cytoplasm into mitochondria in order to stimulate apoptosis. These data suggested that $\mathrm{Ku} 70$ is an inhibitor of Bax-dependent apoptosis. It was hypothesized that the knockdown of $\mathrm{Ku} 70$ may increase TSA-induced apoptosis. In the present study, Ku70 siRNA was used to knockdown Ku70 expression in CRC cells followed by treatment with TSA after $24 \mathrm{~h}$. However, flow cytometric and western blot analyses revealed a decrease in TSA-induced apoptosis following the downregulation of $\mathrm{Ku} 70$.

$\mathrm{Ku}$ proteins, including $\mathrm{Ku} 70$ and $\mathrm{Ku} 80$, are subunits of the DNA-dependent protein kinase (DNA-PK) complex, which are essential for the DNA-binding activity of the complex following DNA breakage in order to facilitate the DNA repair process (16-18). It was previously demonstrated that cells which were defective in any of the DNA-PK subunits, including DNA-PK catalytic subunits Ku70 and Ku80, were highly sensitive to DNA damage as they were unable to repair DNA double-strand breaks efficiently (19). Therefore, the role of $\mathrm{Ku} 70$ has been linked to cell survival and carcinogenesis (20). The natural product justicidin A was reported to induce the apoptosis of CRC cells through decreasing cytoplasmic Ku70 and increasing the mitochondrial translocation of Bax (21). In addition, Ku70 depletion was reported to increase sensitivity to $\mathrm{x}$-ray and radiation-induced caspase-dependent apoptosis in lung cells (22). However, the results of the present study demonstrated that decreased Ku70 levels may impair rather than promote Bax-dependent apoptosis induced by TSA, therefore suggesting that $\mathrm{Ku} 70$ has a complex role in the regulation of apoptosis.

In order to elucidate the reason why $\mathrm{Ku} 70$ knockdown impaired TSA-induced apoptosis in the present study, Bax protein levels were detected by western blot analysis, the results of which revealed decreased Bax expression accompanied with decreased Ku70 expression. This therefore indicated that the decreased stability of the Bax protein occurred as a result of Ku70 depletion. A previous study reported that Bax was ubiquitylated and that $\mathrm{Ku} 70$ promoted the deubiquitylation of Bax (23). Therefore, in the present study, HCT116 CRC cells were treated with the proteosomal inhibitor MG132 following Ku70 siRNA transfection, but prior to TSA treatment. The results showed that MG132 was able to rescue the decreased Bax expression and apoptosis, which were induced by Ku70 knockdown prior to TSA treatment. This therefore suggested 
that $\mathrm{Ku} 70$ had an important role in maintaining the stability of the Bax protein and Bax-initiated apoptosis.

In conclusion, the results of the present study demonstrated that $\mathrm{Ku} 70$ acetylation mediated Bax-dependent apoptosis, which was induced by TSA treatment in CRC cells. This therefore indicated that $\mathrm{Ku} 70$ was essential for the protection of Bax from proteosomal degradation.

\section{Acknowledgements}

The present study was supported by grants from the National Natural Science Foundation of China (nos. 31171344, 81272587 and 81172223) and the General Financial Grant from the China Postdoctoral Science Foundation (no. 2013M542505).

\section{References}

1. Jernal A, Siegel R, Ward E, et al: Cancer statistics, 2009. CA Cancer J Clin 59: 225-249, 2009.

2. Johnstone RW: Histone-deacetylase inhibitors: novel drugs for the treatment of cancer. Nat Rev Drug Discov 1: 287-299, 2002.

3. Yu X, Guo ZS, Marcu MG, et al: Modulation of p53, ErbB1, ErbB2 and Raf-1 expression in lung cancer cells by depsipeptide FR901228. J Natl Cancer Inst 94: 504-513, 2002.

4. Zhu WG and Otterson GA: The interaction of histone deacetylase inhibitors and DNA methyltransferase inhibitors in the treatment of human cancer cells. Curr Med Chem Anti-Cancer Agents 3 : 187-199, 2003.

5. Meinke PT and Liberator P: Histone deacetylase: a target for antiproliferative and antiprotozoal agents. Curr Med Chem 8: 211-235, 2001

6. Yoshida M, Kijima M, Akita M and Beppu T: Potent and specific inhibition of mammalian histone deacetylase both in vivo and in vitro by trichostatin A. J Biol Chem 265: 17174-17179, 1990.

7. Meng J, Zhang HH, Zhou CX, Li C, Zhang F and Mei QB: The histone deacetylase inhibitor trichostatin A induces cell cycle arrest and apoptosis in colorectal cancer cells via p53-dependent and -independent pathways. Oncol Rep 28: 384-388, 2012.

8. Sawada M, Sun W, Hayes P, Leskov K, Boothman DA and Matsuyama S: Ku70 suppresses the apoptotic translocation of Bax to mitochondria. Nat Cell Biol 5: 320-329, 2003.
9. Cohen HY, Lavu S, Bitterman KJ, et al: Acetylation of the C terminus of Ku70 by CBP and PCAF controls Bax-mediated apoptosis. Mol Cell 13: 627- 638, 2004.

10. Zhang F, Bäumer N, Rode M, et al: The inhibitor of growth protein 5 (ING5) depends on INCA1 as a co-factor for its antiproliferative effects. PLoS One 6: e21505, 2011.

11. Kroemer G: Mitochondrial implication in apoptosis: towards an endosymbiont hypothesis of apoptosis evolution. Cell Death Differ 4: 443-456, 1997.

12. Reed JC: Cytochrome c: can't live with it-can't live without it. Cell 91: 559-562, 1997.

13. Lindemann RK, Gabrielli B and Johnstone RW: Histone-deacetylase inhibitors for the treatment of cancer. Cell Cycle 3: 779-788, 2004.

14. De Giorgi F, Lartigue L, Bauer MK, et al: The permeability transition pore signals apoptosis by directing Bax translocation and multimerization. FASEB J 16: 607-609, 2002.

15. Subramanian C, Hada M, Opipari AW Jr, Castle VP and Kwok RP: CREB-binding protein regulates Ku70 acetylation in response to ionization radiation in neuroblastoma. Mol Cancer Res 11: 173-181, 2013.

16. Collis SJ, DeWeese TL, Jeggo PA, et al: The life and death of DNA-PK. Oncogene 24: 949-961, 2005.

17. He F, Li L, Kim D, et al: Adenovirus-mediated expression of a dominant negative Ku70 fragment radiosensitizes human tumor cells under aerobic and hypoxic conditions. Cancer Res 67: 634-642, 2007.

18. Shintani S, Mihara M, Li C, et al: Up-regulation of DNAdependent protein kinase correlates with radiation resistance in oral squamous cell carcinoma. Cancer Sci 94: 894-900, 2003.

19. Hashimoto M, Rao S, Tokuno O, et al: DNA-PK: the major target for wortmannin-mediated radiosensitization by the inhibition of DSB repair via NHEJ pathway. J Radiat Res 44: 151-159, 2003.

20. Com E, Lagadec C, Page A, et al: Nerve growth factor receptor TrkA signaling in breast cancer cells involves Ku70 to prevent apoptosis. Mol Cell Proteomics 6: 1842-1854, 2007.

21. Lee JC, Lee CH, Su CL, et al: Justicidin A decreases the level of cytosolic Ku70 leading toapoptosis in human colorectal cancer cells. Carcinogenesis 26: 1716-1730, 2005.

22. Koike M, Yutoku Y and Koike A: The defect of Ku70 affects sensitivity to $\mathrm{X}$-ray and radiation-induced caspase-dependent apoptosis in lung cells. J Vet Med Sci 75: 415-420, 2013.

23. Amsel AD, Rathaus M, Kronman N and Cohen HY: Regulation of the proapoptotic factor Bax by Ku70-dependent deubiquitylation. Cell Biol 105: 5117- 5122, 2008. 